\title{
Experimental Evidence of Coherence Resonance in an Optical System
}

\author{
Giovanni Giacomelli \\ Istituto Nazionale di Ottica, Largo E. Fermi 6, 50125 Firenze, Italy \\ Massimo Giudici and Salvador Balle \\ Departamento de Física Interdisciplinar, Instituto Mediterraneo de Estudios Avanzados (CSIC-UIB), \\ 07071 Palma de Mallorca, Spain \\ Jorge R. Tredicce \\ Institut Non-Linéaire de Nice, UMR 6618 Centre National de la Recherche Scientifique-Université de Nice Sophia-Antipolis, \\ 06560 Valbonne, France \\ (Received 13 July 1999)
}

\begin{abstract}
Experimental evidence of coherence resonance in an optical system is reported. We show that the regularity of the excitable pulses in the intensity of a laser diode with optical feedback increases when adding noise, up to an optimal value of the noise strength. Both phase and amplitude fluctuations of the pulses play a relevant role in the dynamics of the system. We introduce the joint entropy of the two variables to generalize the indicator of coherence, and we put in evidence the mechanism of destruction of the excitable orbit after the resonance.
\end{abstract}

PACS numbers: 42.65.Sf, 05.40.Ca, 42.55.-f

Noise is usually perceived as a limiting factor for dynamical systems. Indeed, in the largest variety of devices, the increase of the noise amplitude leads to a degradation of the output signal. In nonlinear systems this is not necessarily true; it has been shown that a finite amount of noise may induce a dynamical state which is, according to some indicator, more "ordered." Examples of such behavior are, for instance, the enhancement of the decay time of a metastable state (noise enhanced stability) [1], the synchronization with a weak periodic input signal (stochastic resonance) [2], and the formation of convective structures in spatially extended systems (noise-sustained structures) [3]. The occurrence of noise-ordered behaviors is of primary importance not only in Physics but in almost all real, nonisolated systems where the environment acts as a thermal bath.

Recently, Pikovsky and Kurths studied in Ref. [4] the effect of noise in the Fitz Hugh-Nagumo model of an autonomous excitable oscillator. They showed that the fluctuations of the time between successive excitable pulses are minimized for a well-defined amount of the input noise. They named such a behavior coherence resonance. Excitable systems are relevant in almost every field of science [5], including chemistry (Belousov-Zabotinskii reaction), biology (cardiac tissue, neurons), and physics (liquid crystals, optical systems). The physical meaning of coherence resonance can be understood by considering the features of excitable systems, namely, the pulse firing occurs only when a perturbation overcomes a certain (nonzero) threshold and thereafter the response of the system is almost independent of the size of the perturbation itself (see, e.g., Ref. [6]). The time between consecutive pulses $T$ can be decomposed into an activation time $t_{a}$, which is the time required for the system to escape from the quiescent state, plus a refractory time $t_{r}$, which represents the time necessary for the system to return to the quiescent state after a departure. Therefore, $t_{r}$ is a time fixed by the dynamical structure of the system, while $t_{a}$ depends on the perturbation. This renders the role of noise in the dynamics of an excitable medium highly nontrivial: excitable pulses can be noise induced if the noise amplitude in the system is strong enough. When the firing process is initiated by noise, the statistical properties of the time $t_{a}$ follow Kramer's law [7,8]. For increasing noise amplitude, the average value of $t_{a}$ decreases until it is negligible compared to $t_{r}$, while the orbit corresponding to the excitable pulse is negligibly affected. As a consequence, the pulsing becomes almost periodic with $T \approx t_{r}$ and the coherence of the system has a maximum. Further increase of the noise amplitude induces the deformation of the orbit of the excitable pulses and eventually the dynamics is totally ruled by the noise. We stress that, at the maximum of coherence, the period of the signal is determined by an internal time scale and not by an external modulation as in stochastic resonance.

In this paper, we present an experimental investigation of the effect of noise in an excitable optical system, namely, a semiconductor laser with optical feedback [8,9]. Changing the amount of noise, and choosing the adequate indicators, we report experimental evidence of coherence resonance.

We consider [9] a semiconductor laser subject to optical feedback from an external mirror placed $36 \mathrm{~cm}$ away. The optical feedback reduces the laser threshold by $12 \%$. The laser current is set at $1 \%$ above the solitary laser threshold, and to this cw current we superpose an electrical, broadband ( $>1 \mathrm{GHz})$, Gaussian noise whose rms amplitude can be externally controlled. The laser output is detected by 

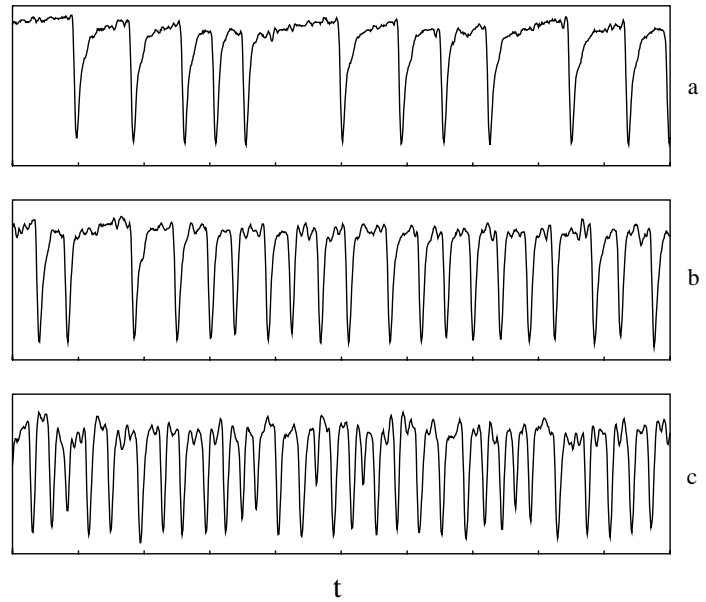

FIG. 1. Temporal behavior of the laser intensity for increasing input noise amplitude. From top to bottom: noise $=-60.8 \mathrm{dBm} /$ $\mathrm{MHz}$ (a), $-52.5 \mathrm{dBm} / \mathrm{MHz}$ (b), and $-44.3 \mathrm{dBm} / \mathrm{MHz}$ (c). The horizontal scale is $100 \mathrm{~ns} /$ div. The vertical scale is the same for the three plots.

a fast avalanche photodiode ( $2 \mathrm{GHz}$ bandwidth) and a LeCroy digital oscilloscope (500 $\mathrm{MHz}$ bandwidth). A DC-100 MHz amplifier allows selection of the frequency range of interest. It is worth noting that filtering the signal at $100 \mathrm{MHz}$ allows us to monitor the excitable pulses without the details introduced by faster time scales. The pulse statistics are obtained from intensity traces containing several thousands of excitable pulses.

In the absence of added noise, the output intensity is constant in time. As the noise is introduced in the system, we observe the appearance of excitable pulses, randomly distributed in time (Fig. 1a). Increasing the amount of noise, the pulse rate increases, until the signal becomes almost periodic (Fig. 1b). For higher noise the signal becomes irregular (Fig. 1c). This behavior is confirmed by the power spectra of the laser intensity shown in Fig. 2, since the spectrum of the trace in Fig. 1b exhibits a peak narrower than those in the power spectra of traces Figs. 1a and 1c. Therefore, the behavior of the system is more

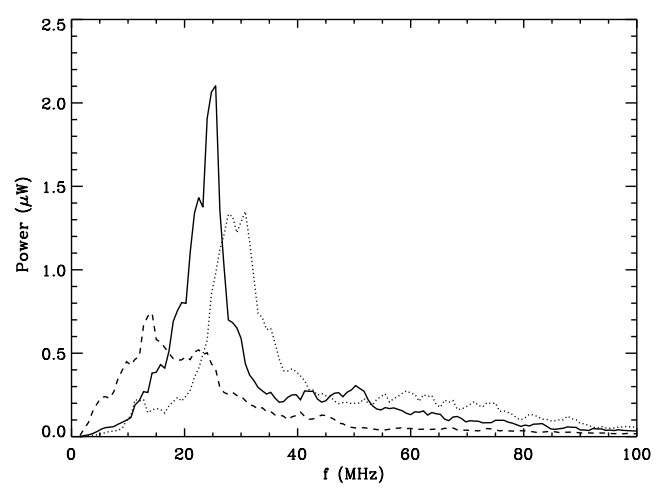

FIG. 2. Power spectrum of each of the traces in Fig. 1. Dashed line: trace (a); solid line: trace (b); and dotted line: trace (c). regular around some definite noise level, as expected from coherence resonance.

Following Ref. [4], we study the behavior of the time interval between pulses $T$ using the indicator $R_{\theta}$, defined as the standard deviation of the scaled variable $\theta=T /\langle T\rangle$. In the case of a perfectly periodic signal, $R_{\theta}$ would be identically zero. In Fig. 3 we plot $R_{\theta}$ as a function of the noise amplitude. We define the pulse time as the instant when the signal crosses, with negative slope, a fixed threshold value corresponding to $80 \%$ of the amplitude of the pulses shown in Fig. 1a. As noise is increased from its minimum value; $R_{\theta}$ reaches a minimum for $\xi=\xi_{\text {opt }}$ and increases afterwards. The minimum is clearly observable for a wide range of threshold choices; the particular threshold value affects only how fast $R_{\theta}$ increases for noise levels above $\xi_{\text {opt }}$, but it does not modify the statistics for noise levels below this optimum value. This is clear evidence of coherence resonance, since near a particular level of input noise we find maximum regularity of the pulse train.

However, it should be noted that there are, in general, two different ways to initiate the degradation of the coherence of a spiking signal. In the first place, noise may induce a large jitter of the time between spikes but leave their amplitude almost unchanged. Another possibility is that large pulse amplitude fluctuations develop as induced by noise. In fact, a simple qualitative analysis of the signal shown in Fig. 1 reveals that, for $\xi>\xi_{\text {opt }}$, noise strongly affects not only the time $T$ between pulses, but also the pulse amplitude $A$. This explains why the choice of threshold value affects $R_{\theta}$ for large noise levels, but it also allows us to explore the mechanisms of degradation of coherence in our system, since deeper insight into the breaking of regularity can be achieved by separating pulse timing (phase) from pulse amplitude.

We define a new indicator $R_{\alpha}$ corresponding to the normalized pulse amplitude $\alpha=A /\langle A\rangle$, and we plot it in

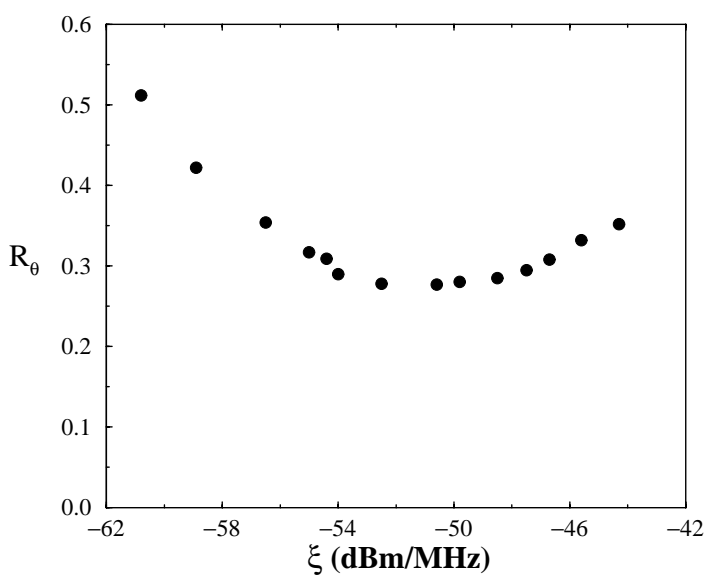

FIG. 3. $R_{\theta}$ as a function of the input noise amplitude. The statistics is performed on pulses whose amplitude cross, with negative slope, a fixed threshold value of $80 \%$, the average amplitude of the pulses shown in Fig. 1a. 


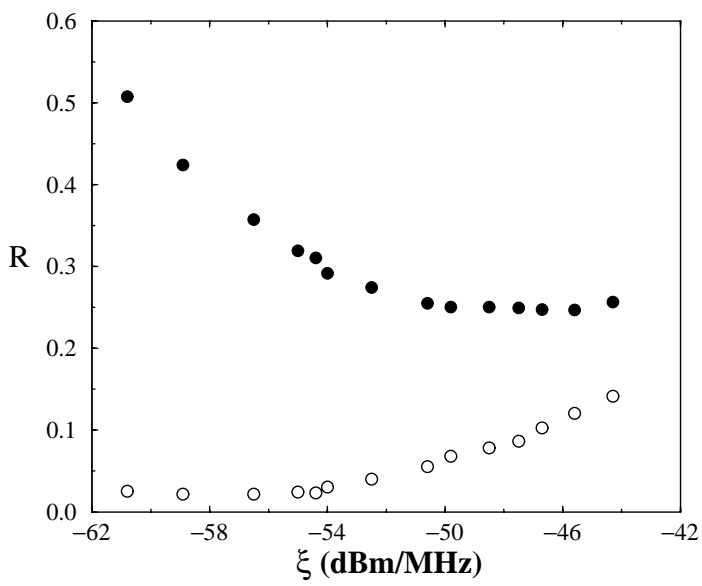

FIG. 4. $\quad R_{\theta}$ (filled circles) and $R_{\alpha}$ (empty circles) as a function of the input noise amplitude. The statistics is performed on pulses whose amplitude cross, with negative slope, a threshold value of $50 \%$ of the average amplitude of the pulses calculated for each noise level.

Fig. 4 together with $R_{\theta}$ as a function of noise level. In order to consider all excitable pulses even for noise levels such that the pulse amplitudes are strongly affected by noise, the threshold value for each noise level has been taken as $50 \%$ of the average signal, i.e., it is changing for different noise levels. In this way, even pulses with relatively small amplitude are considered in the statistics, and we achieve a real separation of phase and amplitude fluctuations of the pulses. In Fig. 3, the same threshold value was considered for all noise levels, thus pulse amplitude fluctuations were translated into time fluctuations that led to an increase in $R_{\theta}$ for noise levels above $\xi_{\text {opt }}$. As can be seen in Fig. 4, with the new choice of threshold, $R_{\theta}$ is initially decreasing and finally becomes almost constant for large noise levels. $R_{\alpha}$ exhibits the opposite behavior: initially it is almost constant, but increases steadily when $R_{\theta}$ becomes nearly constant.

The above results indicate that coherence is degraded more by increasing fluctuations of the pulse amplitudes $A$ than by jitter in the pulse timing. Further confirmation of this mechanism is achieved by considering the joint statistics of the two variables $\alpha$ and $\theta$. We evaluate the joint probability distribution $P(\theta, \alpha)$ from experimental data (Fig. 5). For low noise (Fig. 5a), we observe large temporal fluctuations, but quite a uniform distribution of the pulse amplitudes. By increasing the noise (Figs. 5b and $5 \mathrm{c}$ ), the temporal spread is strongly reduced, but there is a large increase of the fluctuations in $\alpha$ for low temporal separation $T$, indicating that closer pulses exhibit larger amplitude fluctuations.

A quantitative assessment of the above statement is provided by considering the entropy

$$
H=-\sum P \log P,
$$

which is a measure of the degree of disorder in the system (see, e.g., Refs. [10,11], and references therein).

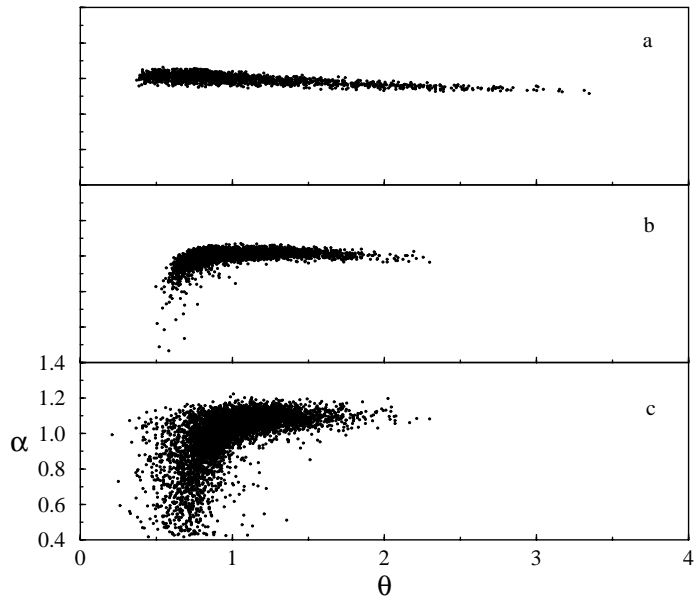

FIG. 5. Distributions of the $(\theta, \alpha)$ values. The coordinates of each point are rescaled to the corresponding averages. The plots correspond, from top to bottom, to the measurements reported in Fig. 1.

For two variables it is found that $H(x, y) \leq H(x)+$ $H(y)$, where the equality holds if, and only if, $x$ and $y$ are independent variables. In such a case, and if the variables are Gaussian, it is found that [11]

$$
\exp [H(x, y)]=2 \pi e \times R_{x} R_{y} .
$$

The quantity $H(x, y)$ then measures the spreading of the (bidimensional) probability distribution and, therefore, it is a meaningful way of describing the total amount of fluctuations of the system. Therefore, the joint entropy $H(\theta, \alpha)$ is a generalized indicator of coherence resonance that takes into account the fluctuations in both time and amplitude of the excitable pulses. We plot $H(\theta, \alpha)$ as a function of the noise added into the system in Fig. 6. It can be seen that the minimum appears again, thus evidencing that there is a noise level that yields maximum regularity in the pulse train.

Finally, it is worth mentioning that, although $H(\theta, \alpha)$ is initially decreasing with noise level, the entropy for the

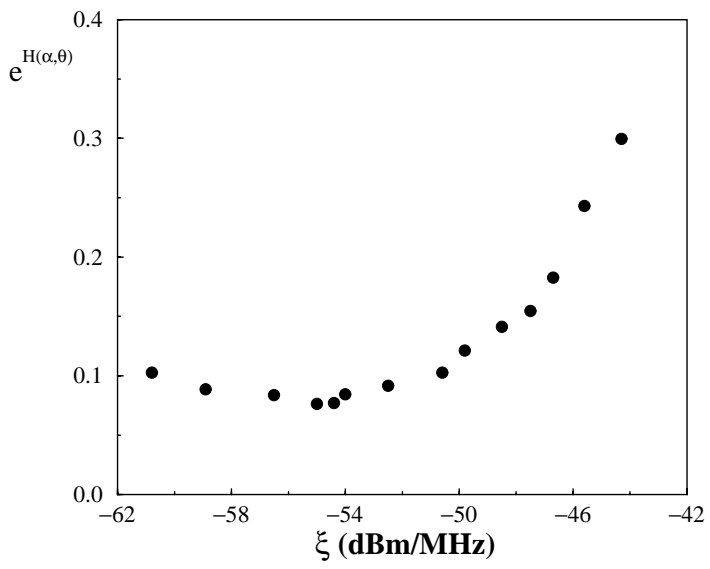

FIG. 6. Joint entropy of the excitable pulses, $H(\theta, \alpha)$ as a function of the input noise level. 
whole data series is monotonically increasing for increasing noise levels. Only the entropy corresponding to the pulse train reveals the coherence resonance effect.

In conclusion, we have shown experimentally the existence of coherence resonance in an optical system. We characterized it by using different statistical indicators: the normalized standard deviation of the time intervals between pulses and the joint entropy of the pulse amplitudes and pulse time interval distributions. We are then able to reveal the specific mechanism for which noise destroys the coherence of the pulse train after the resonance.

We wish to thank A. Politi, E. Hernández-García, M. Zimmermann, O. Piro, and M. San Miguel for very useful discussions. G. G. acknowledges partial support from Contract No. 96.00267.CT02 of the Italian National Council of Research (CNR). M. G. and S. B. acknowledge financial support from Comisión Interministerial de Ciencia y Tecnología under Projects No. TIC98-0418-C05-02 and No. PB94-1167. J.R. T. acknowledges support from Esprit Contract PIANOS.

[1] R. N. Mantegna and B. Spagnolo, Phys. Rev. Lett. 76, 563 (1996).

[2] R. Benzi, A. Sutera, and A. Vulpiani, J. Phys. A 14, 453 (1981); C. Nicolis and G. Nicolis, Tellus 33, 225 (1981); for a review, see, e.g., L. Gammaitoni, P. Hänggi, P. Jung, and F. Marchesoni, Rev. Mod. Phys. 70, 223 (1998); recent evidence in optics has been reported in G. Giacomelli, F. Marin, and I. Rabbiosi, Phys. Rev. Lett. 82, 675 (1999).

[3] M. Santagiustina, P. Colet, M. San Miguel, and D. Walgraef, Phys. Rev. Lett. 79, 3633 (1997).

[4] A. Pikovsky and J. Kurths, Phys. Rev. Lett. 78, 775 (1997).

[5] R. FitzHugh, Biophys. J. 1, 445 (1961); J. Nagumo, S. Arimoto, and S. Yoshizawa, Proc. IREE Aust. 50, 2061 (1962); V. I. Krinsky, in Self Organization, Autowaves and Structures far from Equilibrium, edited by V.I. Krinsky (Springer, Berlin, 1984); A. M. Zhabotinskii, Concentration Autooscillations (Nauka, Moscow, 1974); T. Frisch, S. Rica, P. Coullet, and J. M. Gilli, Phys. Rev. Lett. 72, 1471 (1994); P. Coullet, D. Daboussy, and J. R. Tredicce, Phys. Rev. E 58, 5347 (1998).

[6] P. Coullet, T. Frisch, J. M. Gilli, and S. Rica, Chaos 4, 485 (1994).

[7] H. A. Kramers, Physica (Utrecht) 7, 284 (1940).

[8] M. C. Eguia, G. B. Mindlin, and M. Giudici, Phys. Rev. E 58, 2636 (1998).

[9] M. Giudici, C. Green, G. Giacomelli, U. Nespolo, and J. R. Tredicce, Phys. Rev. E 55, 6414 (1997).

[10] R. Badii and A. Politi, Complexity: Hierarchical Structures and Scaling in Physics (Cambridge University Press, Cambridge, 1997).

[11] A. Papoulis, Probability, Random Variables and Stochastic Processes (McGraw-Hill, Singapore, 1984), 2nd ed. 